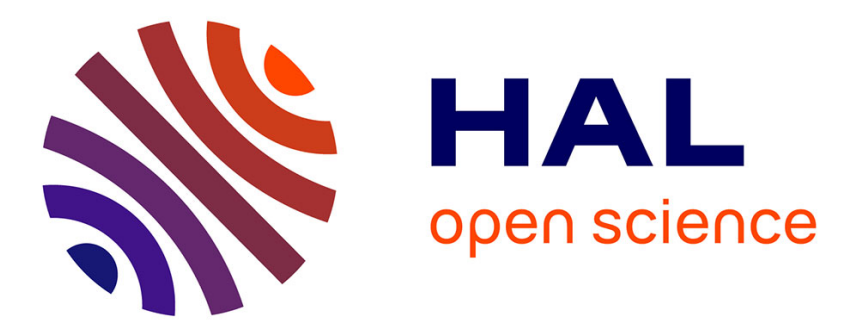

\title{
Molecular Quadrupole Moments Promote Ground-State Charge Generation in Doped Organic Semiconductors
}

\author{
Alberto Privitera, Giacomo Londi, Moritz Riede, Gabriele d'Avino, David \\ Beljonne
}

\section{- To cite this version:}

Alberto Privitera, Giacomo Londi, Moritz Riede, Gabriele d'Avino, David Beljonne. Molecular Quadrupole Moments Promote Ground-State Charge Generation in Doped Organic Semiconductors. Advanced Functional Materials, 2020, 30 (45), pp.2004600. 10.1002/adfm.202004600 . hal-03011441

\author{
HAL Id: hal-03011441 \\ https://hal.science/hal-03011441
}

Submitted on 3 Dec 2020

HAL is a multi-disciplinary open access archive for the deposit and dissemination of scientific research documents, whether they are published or not. The documents may come from teaching and research institutions in France or abroad, or from public or private research centers.
L'archive ouverte pluridisciplinaire HAL, est destinée au dépôt et à la diffusion de documents scientifiques de niveau recherche, publiés ou non, émanant des établissements d'enseignement et de recherche français ou étrangers, des laboratoires publics ou privés. 


\section{Molecular quadrupole moments facilitate ground-}

\section{state charge generation in doped organic}

\section{semiconductors}

Alberto Privitera, ${ }^{I \pm}$ Giacomo Londi, ${ }^{2 \pm}$ Moritz Riede, ${ }^{l}$ Gabriele D'Avino,${ }^{3}$ David Beljonne ${ }^{2 *}$

${ }^{ \pm}$These authors contributed equally

${ }^{1}$ Clarendon Laboratory, Department of Physics, University of Oxford, Oxford OX1 3PU, UK

${ }^{2}$ Laboratory for Chemistry of Novel Materials, University of Mons, B-7000 Mons, Belgium

${ }^{3}$ Institut Néel, CNRS and Grenoble Alpes University, F-38042 Grenoble, France

*email: david.beljonne@umons.ac.be 
ABSTRACT. We have investigated the role of local environmental interactions on the generation of free charge carriers in doped organic layers. Via a self-consistent micro-electrostatic model, we demonstrate the dual effect of molecular quadrupole moments of host and dopant molecules on doping. Namely, electrostatic interactions ease ionization of the dopant by altering the energy level alignment between the host and the dopant and reduce the barrier for charge dissociation by flattening the energy landscape around the ionized dopants. These results indicate that tailoring molecular quadrupole moments of the host and/or dopant is an attractive strategy towards improved doping efficiency in organic semiconductors.

KEYWORDS Molecular doping, electron affinity, ionization potential, charge generation, quadrupolar moment, organic semiconductors

\section{TOC GRAPHICS}

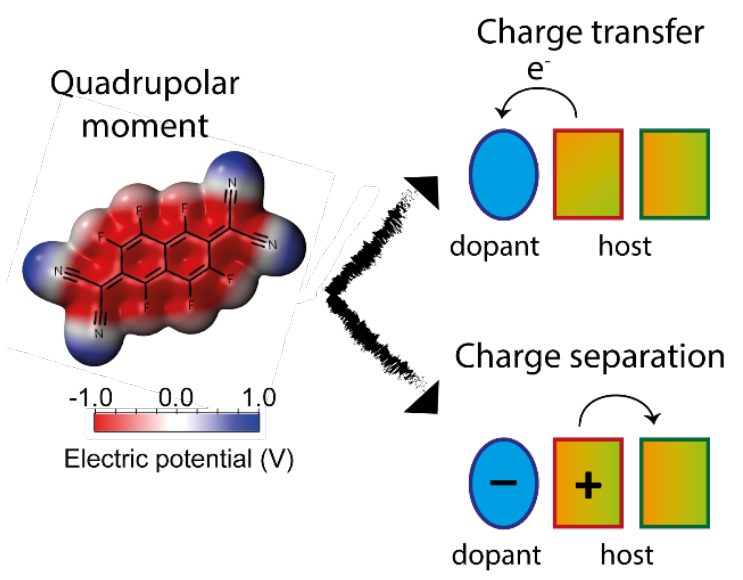


NEW CONCEPTS. Organic semiconductors are emerging as a viable alternative to their inorganic counterparts in optoelectronic applications such as organic LEDs, organic solar cells, organic FETs and sensing devices. Despite molecular doping contributed to this success, namely by allowing fine tuning of the Fermi level in stacked devices, the doping efficiency in organic semiconductors is still very low, sometimes in the range of only a few percent. In this communication, we demonstrate that the molecular quadrupole moments of the host and the dopant both strongly affect the charge generation mechanism in doped organic layers. Our detailed micro-electrostatic calculations show that a careful engineering of the molecular quadrupole moments can strongly favor the dopant ionization mechanism by rearranging the frontier energy levels of the host/dopant couples and improve charge dissociation by decreasing the effective electron-hole Coulomb energy barrier. 
Molecular doping has proved to be a game-changer in the field of organic optoelectronics as it allows for example the precise alignment of the energy levels at the interfaces of multi-layer devices and the minimization of Ohmic losses at the electrodes..$^{1-4}$ As a result, molecular doping has laid the foundations for the development of efficient organic devices including organic light emitting diodes (OLEDs), organic field effect transistors (OFETs) and organic solar cells (OSCs).5-

${ }^{7}$ Despite the significant technological impact of molecular doping, we are still currently lacking a detailed understanding of its mechanisms,${ }^{89}$ which has precluded the identification of molecular design strategies and has led to disappointing charge generation efficiency in doped organic layers. ${ }^{10}$

What is currently known is that charge generation in doped organic semiconductors consists of two elementary steps. ${ }^{2,10,11}$ The first step involves either a hybridization of the host-dopant frontier molecular orbitals or a ground-state integer charge transfer $(\mathrm{CT})$ process between the host and the dopant. ${ }^{1,12,13}$ For p-type doping, the latter is favoured when the energy difference between the ionization potential (IP) of the host and the electron affinity (EA) of the dopant (i.e. the host-dopant gap $\left.\Gamma_{h d}=I P_{\text {host }}-E A_{d o p}\right)$ is smaller than the Coulomb binding energy $\left(V_{e h}\right)$ between an electron and its geminate hole sitting on nearest neighbour molecules. ${ }^{14,15}$ In such conditions, a bound CT state between the hole left on the host and the electron added on the dopant is generated..$^{11,12}$ This is the ionization step. The second step, namely the charge dissociation, then consists of the spatial migration of the hole (for p-doping) away from the ionized acceptor that requires overcoming the large $V_{e h}$ Coulomb binding of the CT pair, which usually amounts to several hundreds of meV. ${ }^{16-}$ ${ }^{18}$ The energetic and kinetic aspects of both steps need to be concomitantly optimized in order to maximize the overall charge generation efficiency, which calls for a control of the interactions between the molecules at the microscopic level. ${ }^{10}$ 
Tuning these microscopic interactions can be achieved by molecular and material engineering. For instance, Ross et al. demonstrated that the energy levels in an organic semiconductor and the Fermi energy can be simultaneously tuned by taking advantage of molecular quadrupolar interactions. ${ }^{3}$ This result was achieved by co-evaporating a host mixture between zincphthalocyanine $(\mathrm{ZnPc})$ and its eight-times fluorinated counterpart $\left(\mathrm{F}_{8} \mathrm{ZnPc}\right)$ together with the ptype dopant F6-TCNNQ. Such mixtures are very interesting because they provide an effective and practical approach to finely control the p-doping efficiency of F6-TCNNQ upon tuning the host energy levels, which are a function of the $\mathrm{ZnPc}: \mathrm{F}_{8} \mathrm{ZnPc}$ molar ratio., ${ }^{3,19,20}$

Here, in the wake of the very recent experimental work reported by Ross et al., ${ }^{3}$ we investigate the role of environmental interactions on the charge generation mechanism in doped binary (ZnPc:F6-TCNNQ and $\mathrm{F}_{8} \mathrm{ZnPc:F6-TCNNQ)}$ ) and ternary (ZnPc:F8nPc:F6-TCNNQ) blends. Based on an in-depth atomistic modelling of electrostatic and dielectric phenomena in molecular solids, ${ }^{21}$ we show that charge-quadrupole interactions affect both the ionization step (by reshuffling the energy levels of the dopant and the host) and the charge dissociation step (by creating a favourable energy pathway for the hole). Interestingly, although the solid-state interactions are commonly considered long-range, we observe that the substitution of a host molecule with F6TCNNQ has a significant short-range effect, namely the IP of the host molecules in closest proximity to the dopant strongly differ by $\sim 0.4 \mathrm{eV}$ from the IPs of the other host molecules. This electrostatic contribution is mainly due to the charge-quadrupolar moment of F6-TCNNQ and significantly affects the $\Gamma_{h d}$ gap, which on average amounts to $0.63 \mathrm{eV}$ for $\mathrm{ZnPc}$ and $0.86 \mathrm{eV}$ for $\mathrm{F}_{8} \mathrm{ZnPc}$. When accounting for electron-hole Coulomb interactions $V_{e h}$, we find an overall energy barrier that is close to zero for the formation of a bound, First Nearest Neighbor (FNN) pair for a binary ZnPc:F6-TCNNQ blend. In $\mathrm{F}_{8} \mathrm{ZnPc:F6-TCNNQ} \mathrm{this} \mathrm{barrier} \mathrm{is} 0.2 \mathrm{eV}$ larger, in line with 
the poorer ionization efficiency observed in literature for the latter blend. Notably, $V_{e h}$ also provides a quantitative estimate of the energy that needs to be overcome to free the charges of the CT state, which turns out to be sizeable in both blends $(\sim 0.65 \mathrm{eV})$. Very interestingly, by extending the calculations beyond FNNs, we also notice that the quadrupolar field generated by the F6TCNNQ dopant positively impacts charge dissociation by considerably reducing this energy barrier. We believe these results pave the way towards a full control of both steps (ionization and charge dissociation) in the doping of organics through the proper engineering of molecular quadrupolar moments.

First, we calculated the gas-phase IP and EA of the host ( $\mathrm{ZnPc}$ and $\left.\mathrm{F}_{8} \mathrm{ZnPc}\right)$ and p-dopant (F6TCNNQ) molecules (see chemical structures in Figure 1a and SI2) at the evGW many-body perturbation theory level. ${ }^{22,23}$ The obtained values, reported in Figure SI3, are in excellent agreement with experimental values,${ }^{24}$ and indicate an increase in both the molecular IP and EA by $\sim 0.5 \mathrm{eV}$ upon fluorination, as a result of the strong, inductive, electron-withdrawing effects induced by the fluorine atoms. ${ }^{25}$ The huge energy difference in the gas-phase between the EA of F6-TCNNQ (4.44 eV) and the IP of the host $\left(6.41 \mathrm{eV}\right.$ for $\mathrm{ZnPc}$ and $6.91 \mathrm{eV}$ for $\left.\mathrm{F}_{8} \mathrm{ZnPc}\right)$ would completely impede ground-state CT. The situation changes dramatically when accounting for environmental solid-state effects on the energy levels of both the host and dopant molecules..21,26 In molecular solids, the IP and EA values depend strongly on collective, long-range, electrostatic effects associated with the varying charge density distributions in the neutral and ionized molecules. These include induction effects, describing the dielectric screening of the polarizable medium to an added charge, and electrostatic interactions, brought about by the molecular electrical multipole moments. ${ }^{21}$ In our case, electrostatic interactions are led by the quadrupole term, since the molecules of interest are centrosymmetric and have no dipole moment. ${ }^{19}$ 
We first consider the case of a F6-TCNNQ dopant as a single substitutional impurity in two pristine lattices, the first made of $\mathrm{ZnPc}$ molecules and the second of $\mathrm{F}_{8} \mathrm{ZnPc}$ ones. We specifically considered films (2D slab calculations) of the two compounds, composed of molecular stacks whose axis lies in the film plane, leading to an edge-on molecular orientation with respect to the slab normal (Figure SI4). ${ }^{19}$ Electrostatic and induction phenomena are described here with a classical Micro-Electrostatic (ME) model of atomistic resolution, see Methods in SI. ${ }^{27}$ Our calculations reveal large environmental effects on charge energetics, as shown in Figures $1 \mathrm{~b}$ and 1c. ${ }^{26}$ In line with earlier findings, we notice that induction interactions reduce the host-dopant gap by $\sim 2 \mathrm{eV}$, irrespective on the host material, as a result of the similar dielectric susceptibilities. ${ }^{21,28}$

On top of induction, the electrostatic contribution further affects the energy levels with an amplitude and sign that depend on the host material. Namely, these interactions are determined by the out-of-plane component of the traceless quadrupolar tensor, which is positive in $\mathrm{ZnPc}$ and negative in $\mathrm{F}_{8} \mathrm{ZnPc}$ because of the inverted polarity of $\mathrm{C}-\mathrm{F}$ vs $\mathrm{C}-\mathrm{H}$ bonds (Table SI1). In Figures $1 \mathrm{~b}$ and 1c, we distinguish between the IP of an host molecule far away from the dopant (open circle) and an host molecule sitting next to the dopant along the $\pi-\pi$ stacking direction (full circle), which we consider the most important for both charge motion and electrostatic interactions. We notice that the sign of the electrostatic shifts of the energy levels of both the dopant and host molecules is the same as the pristine host, which is negative for $\mathrm{ZnPc}$ (Fig. 1a) and positive for $\mathrm{F}_{8} \mathrm{ZnPc}$ (Fig. 1b). This determines a $1 \mathrm{eV}$ difference in the EA between the two hosts, in line with recent findings by some of us. ${ }^{26}$ Interestingly, we also observe that the IP of a host molecule does strongly depend on its position with respect to the dopant. The position dependence of the host levels can be ascribed to the different charge-quadrupolar moment of the dopant with respect to 
the host molecule. Being originated by the quadrupole of the single dopant molecule, this effect is short-range and has an appreciable impact only on the nearest-neighbor molecules.
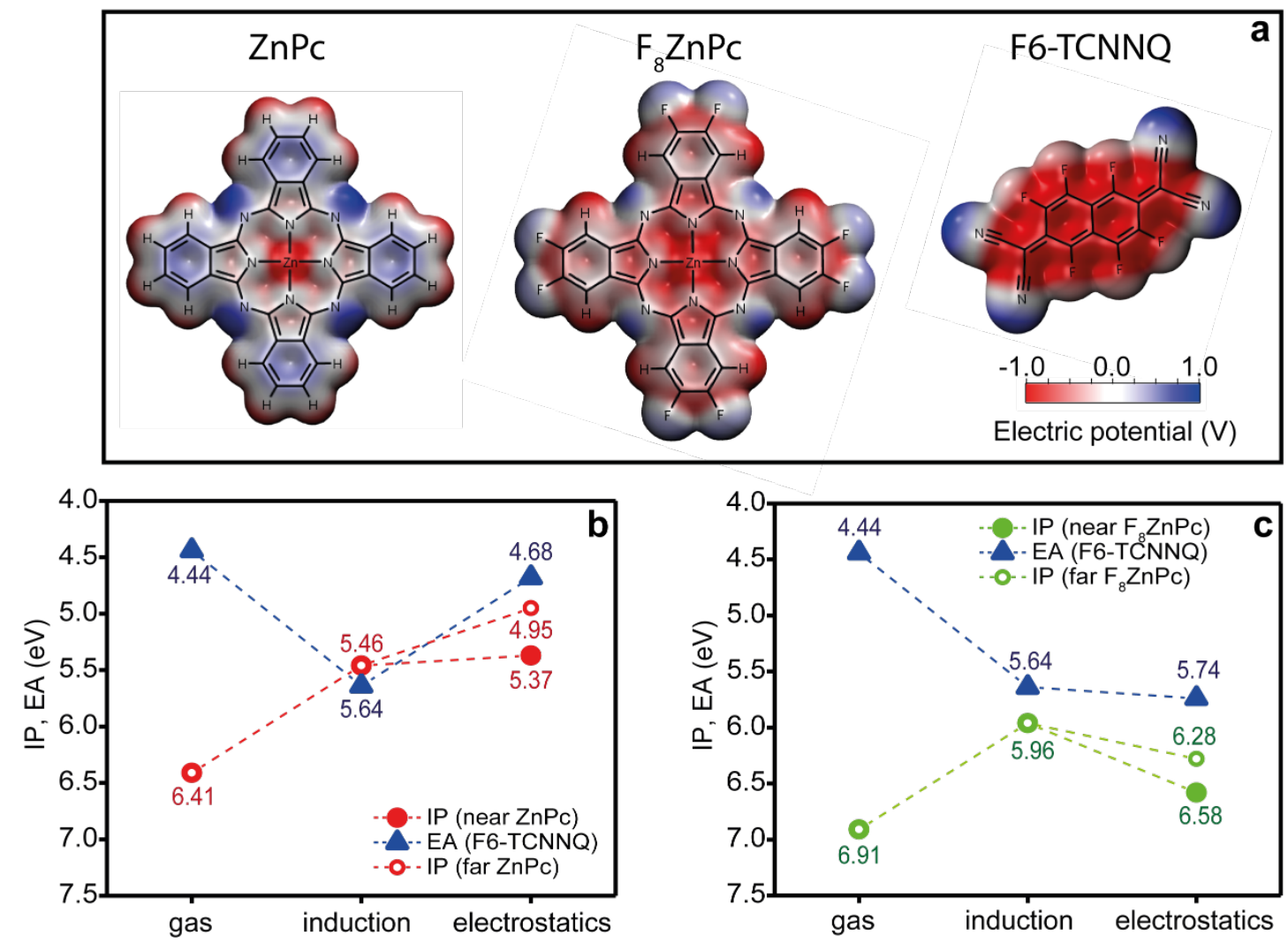

Figure 1 - Binary blends. (1) Molecular structures and renderings of the electrostatic potentials of ZnPc, $\mathrm{F}_{8} \mathrm{ZnPc}$ and F6-TCNNQ, as obtained from DFT calculations at the PBE0/cc-pVTZ level of theory. (b, c) Effect of induction and electrostatic interactions on the frontier energy levels of the dopant (blue triangles) and the host molecules, either in close proximity (full circle) or far away (open circle) from the dopant. Specifically, (b) binary blend composed of ZnPc (host, red dots) and F6-TCNNQ (dopant, blue triangles) and (c) binary blend composed of $\mathrm{F}_{8} \mathrm{ZnPc}$ (host, green dots) and F6-TCNNQ (dopant, blue triangles). 
We now turn our attention to the energy levels of the ternary blends composed by a mixed $\mathrm{ZnPc}: \mathrm{F}_{8} \mathrm{ZnPc}$ host, doped with a single F6-TCNNQ molecule. ME calculations were carried out for different host mixing ratios, ranging from pure $\mathrm{ZnPc}$ to pure $\mathrm{F}_{8} \mathrm{ZnPc}$. For the $1: 1$ ratio, we opt for an alternating $\mathrm{ZnPc}-\mathrm{F}_{8} \mathrm{ZnPc}$ mixed-stack motif, which is expected to be the most stable structure for $\pi$-stacked molecules with opposite quadrupoles. A similar alternating packing was recently resolved for a 1:1 co-crystal of pentacene and perfluoropentacene.${ }^{29}$ Crystalline supercells of blends with different ratio were created in a similar way but with a different alternating periodicity (one $\mathrm{ZnPc}$ molecule every two $\mathrm{F}_{8} \mathrm{ZnPc}$ molecules, and vice versa). Our calculations for the energy levels in undoped $\mathrm{ZnPc}: \mathrm{F}_{8} \mathrm{ZnPc}$ blends of different composition (Figure SI5) are in substantial agreement with the similar ones reported by Schwarze et al. ${ }^{19}$

The energy levels of host and dopant molecules in ternary blends are shown in Figure 2a. Since different microscopic configurations can be generated upon introducing a dopant in the $\mathrm{ZnPc}: \mathrm{F}_{8} \mathrm{ZnPc}$ blends (for the 1:1 ratio, the dopant can be placed both at $\mathrm{ZnPc}$ and $\mathrm{F}_{8} \mathrm{ZnPc}$ site; more configurations are possible for other host composition), the figure displays the average over the possible microstructures, which is representative of the specific values reported in Figure SI6. Calculations in Figure 2a show that the energy levels of host molecules and dopant evolve linearly with the $\mathrm{ZnPc}$ molar ratio, as all the molecules probe an effective quadrupolar field resulting from the additive contributions sourced by the two host molecules. Most importantly, this translates into a $\Gamma_{h d}$ energy difference that does not significantly vary with the host mixing ratio, leading to the conclusion that replacing a significant fraction of $\mathrm{ZnPc}$ molecules by electron-poor $\mathrm{F}_{8} \mathrm{ZnPc}$ should not be overly detrimental to ionization, at least from an energetic point of view. This is an important result that laid down the foundation of the statistical approach successfully used by Ross et al. to model the ternary blends. ${ }^{3}$ 
On average, when accounting for the whole range of host chemical composition, the $\Gamma_{h d}$ gap ranges from $0.63 \mathrm{eV}$ for $\mathrm{ZnPc}$ to $0.86 \mathrm{eV}$ in $\mathrm{F}_{8} \mathrm{ZnPc}$ (Figure $2 \mathrm{~b}$ ). This energy difference is largely compensated by the electron-hole Coulomb binding energy $V_{e h}(\sim 0.65 \mathrm{eV})$, making the overall ionization process barrier-free for $\mathrm{ZnPc}$, while still significantly activated (energy barrier $\sim 0.2 \mathrm{eV}$ ) for $\mathrm{F}_{8} \mathrm{ZnPc}$ (Figure 2b). This is in good agreement with the high ionization efficiency experimentally measured in the ZnPc:F6-TCNNQ blends. ${ }^{3}$ Conversely, CT state energy for the $\mathrm{F}_{8} \mathrm{ZnPc}$ case remains positive and higher than the thermal energy, which underlines the ionization efficiency is low, in line with our previous experimental results. ${ }^{3}$ In addition to $V_{e h}$, we considered the role played by structural relaxations upon charging, ${ }^{26}$ the so-called polaronic effect $\left(\lambda=\lambda^{+}+\right.$ $\left.\lambda^{-}\right)$. This contribution $(\sim 0.15 \mathrm{eV})$ further stabilizes the CT state energy, making the CT process from the host to the dopant even easier (Figure 2b).
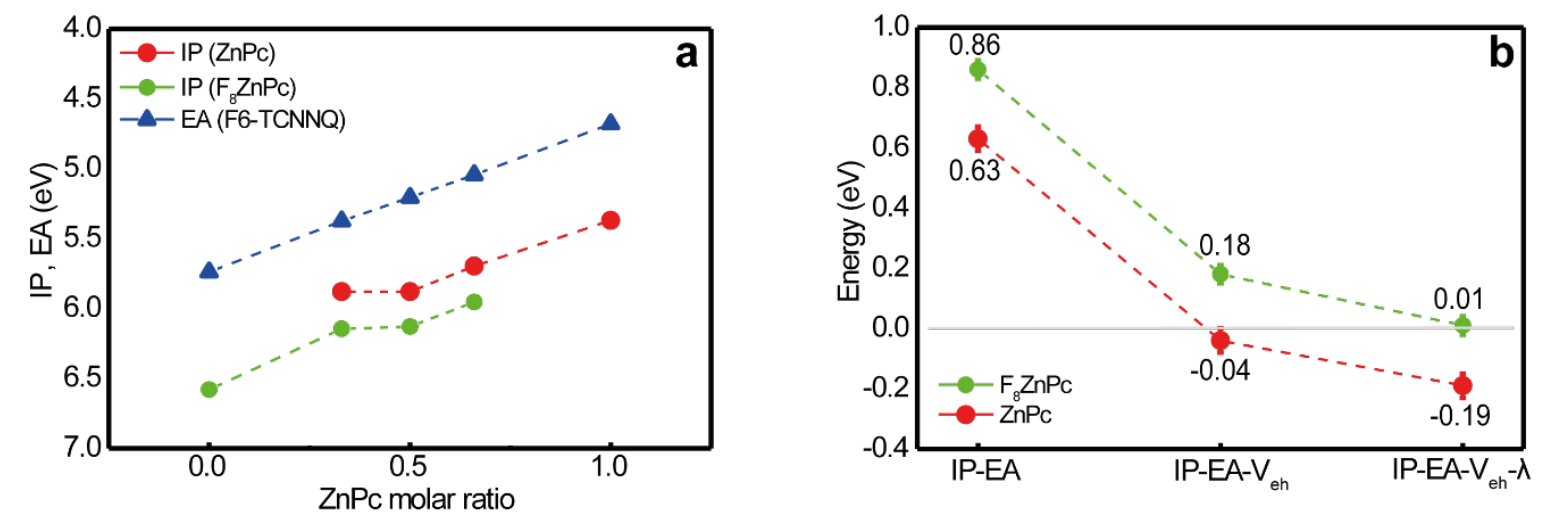

Figure 2 - Ternary blends. (a) Effect of the solid-state interactions on the IPs of ZnPc (red circles) and $\mathrm{F}_{8} \mathrm{ZnPc}$ (green circles) and the EA of F6-TCNNQ (blue triangles) in ZnPc:F8ZnPc blends doped with F6-TCNNQ as a function of $\mathrm{ZnPc}$ molar ratio. The data correspond to averages over different microscopic hosts-dopant configurations. (b) Energies of the CT state where the electron is localized on the F6-TCNNQ and the hole either on the FNN ZnPc or $\mathrm{F}_{8} \mathrm{ZnPc}$. The energy was 
calculated by considering different contributions, namely (1) the $\Gamma_{h d}$ energy difference, (2) the eh Coulomb interaction $\left(V_{e h}\right)$, and (3) the relaxation energy of the CT state $(\lambda)$. The last two contributions stabilize the $\Gamma_{h d}$ energy difference and make the CT state more stable.

Moving on further, we discuss the results of similar calculations performed for molecules from first to fifth neighbour along the $\pi-\pi$ stacking direction, as they are relevant for charge separation of the CT states. In Figure 3 and Table SI5, we report the energies of the CT states and the $V_{e h}$ binding energies as a function of the hole distance from the F6-TCNNQ anion for the ZnPc:F6TCNNQ and $\mathrm{F}_{8} \mathrm{ZnPc}$ :F6-TCNNQ binary blends and for ternary blends with 1:1 ZnPc:F $\mathrm{ZnPc}$ composition, distinguishing the two cases where the dopant sits at the molecular site of $\mathrm{ZnPc}$ or $\mathrm{F}_{8} \mathrm{ZnPc}$. Remarkably, our calculations highlight that the activation energy barrier for charge separation of the CT state is significantly lower than the mere Coulomb binding energy $V_{e h}$, which is $0.65 \mathrm{eV}$ (compare red and black lines in Figure 3). The activation energy reduces to $\sim 0.1 \mathrm{eV}$ in ZnPc:F6-TCNNQ binary systems and is slightly larger $(\sim 0.25 \mathrm{eV})$ in $\mathrm{F}_{8} \mathrm{ZnPc:F6-TCNNQ}$. We explain this massive reduction in effective binding energy between the holes and their ionized acceptors as resulting from the changing quadrupolar field experienced by the host molecules when further away from the F6-TCNNQ dopant. This appears particularly clear when looking at the IPs of the host molecules as a function of the distance from F6-TCNNQ (TableSI4, Figure SI7). The calculations show that the induction effect is similar for all the molecular positions and the main responsible for this energetic rearrangement is the electrostatic contribution. Such an energy level bending effect, previously reported in the context of photoinduced charge separation at donoracceptor interfaces, ${ }^{30-33}$ extends over 3-4 molecules and helps the holes to escape their Coulomb wells. 

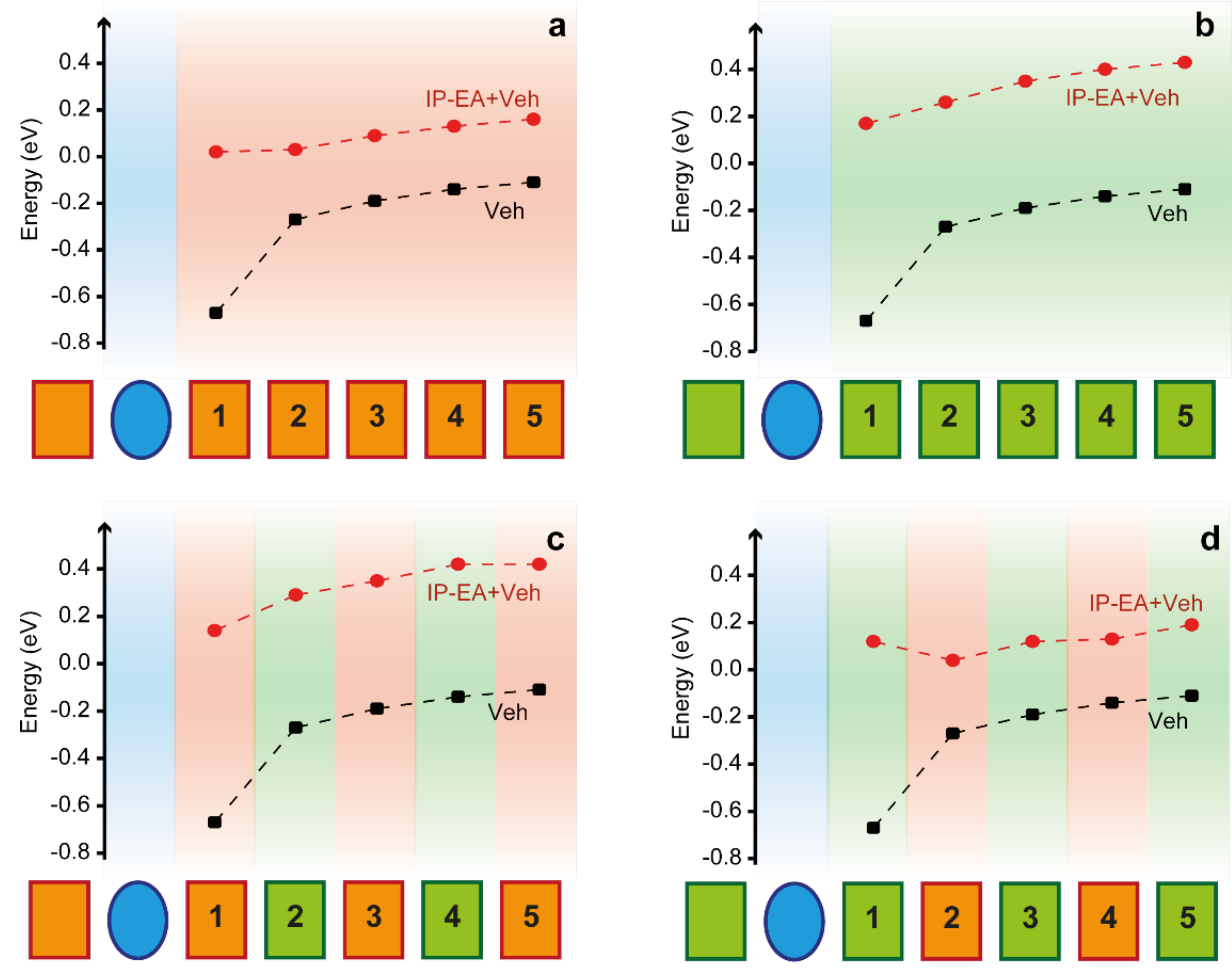

Figure 3 - Charge separation. Energies of the CT state (red dots) and $V_{e h}$ binding energies (black squares) where the electron is localized on the F6-TCNNQ and the hole on either $\mathrm{ZnPc}$ or $\mathrm{F}_{8} \mathrm{ZnPc}$ host molecules, from the first to the fifth nearest neighbours along the $\pi-\pi$ stacking direction. The calculations are reported for (a) ZnPc:F6-TCNNQ and (b) F8ZnPc:F6-TCNNQ binary blends, and two 1:1 configurations of (c,d) ZnPc:F8 $\mathrm{ZnPc}: \mathrm{F6}$-TCNNQ ternary blends. The CT state energy was calculated by considering different contributions, namely (1) the $\Gamma_{h d}$ energy difference, (2) the eh Coulomb interaction $\left(V_{e h}\right)$. Legend: orange square $=\mathrm{ZnPc}$, green square $=\mathrm{F}_{8} \mathrm{ZnPc}$, blue oval $=$ F6-TCNNQ.

In conclusion, our work delved into the effect of the environmental solid-state interactions on the generation of free charge carriers by doping organic semiconductors. We carried out our investigations on a model system comprising $\mathrm{ZnPc}: \mathrm{F}_{8} \mathrm{ZnPc}$ blend, as a host, p-doped with F6- 
TCNNQ. This ternary blend allows to control doping efficiency by simply varying the ZnPc molar ratio. ${ }^{3}$ Our theoretical work disclosed the fundamental role of charge-quadrupolar interactions on both (1) the ionization and (2) the charge dissociation steps of molecular doping. As for the ionization step, our work revealed that the presence of a substitutional F6-TCNNQ molecule in the host lattice strongly influence (by $\sim 0.4 \mathrm{eV}$ ) the IP of the nearest-neighbor molecules without affecting those further apart. This short-range electrostatic contribution significantly affects the $\Gamma_{h d}$ gap, which on average amounts to $0.63 \mathrm{eV}$ for $\mathrm{ZnPc}$ and $0.86 \mathrm{eV}$ for $\mathrm{F}_{8} \mathrm{ZnPc}$. In addition, after considering the electron-hole Coulomb interactions $\left(V_{e h}\right)$, the overall energy barrier for the formation of the CT state between FNN molecules becomes close to zero for ZnPc/F6-TCNNQ pairs and is $\sim 0.2 \mathrm{eV}$ larger for $\mathrm{F}_{8} \mathrm{ZnPc} / \mathrm{F} 6-\mathrm{TCNNQ}$ pairs, in line with the larger ionization efficiency measured in the former blend. The charge dissociation step is expected to be very inefficient on the sole consideration of the Coulomb binding energy of the generated holes to their ionized acceptors, with $V_{e h}$ as large as $\sim 0.65 \mathrm{eV}$. Remarkably, however, this is largely alleviated by longer-range charge-quadrupole interactions that tilt the energy landscape in favor of dissociation, with residual effective energy barriers in the range $0-0.2 \mathrm{eV}$ depending on the local micro-structural configuration.

All in all, our findings highlight that a thorough engineering of molecular quadruple moments of both the host and the dopant is essential to control the ionization and the charge dissociation steps in molecular doping and to develop a new generation of host/dopant couples with improved doping efficiency.

\section{AUTHOR CONTRIBUTION}




\section{ACKNOWLEDGEMENTS}

This work was supported by European Union's Horizon 2020 research and innovation programme under Marie Sklodowska Curie Grant agreement No.722651 (SEPOMO project) and by the EPSRC (EP/L011972/1). Computational resources in Mons were provided by the Consortium des Équipements de Calcul Intensif (CÉCI), funded by the Fonds de la Recherche Scientifiques de

Belgique (F.R.S.-FNRS) under Grant No. 2.5020.11, as well as the Tier-1 supercomputer of the Fedération Wallonie-Bruxelles, infrastructure funded by the Walloon Region under Grant Agreement No. 1117545. D.B. is a FNRS Research Director.

\section{Supporting Information.}

Theoretical details, molecular structures, DFT calculations quadrupole moments, molecular stack, calculated IPs for first and second nearest neighbors in binary ( $\mathrm{ZnPc}: \mathrm{F}_{8} \mathrm{ZnPc}$ ) and ternary (ZnPc:F $\mathrm{ZnPc}: \mathrm{F6}-\mathrm{TCNNQ}$ ) blends and calculated energy levels of the CT state are reported. This information is available free of charge at the ACS Publications website.

\section{REFERENCES}

(1) Salzmann, I.; Heimel, G.; Oehzelt, M.; Winkler, S.; Koch, N. Molecular Electrical Doping of Organic Semiconductors: Fundamental Mechanisms and Emerging Dopant Design Rules. Acc. Chem. Res. 2016, 49, 370-378.

(2) Tietze, M. L.; Benduhn, J.; Pahner, P.; Nell, B.; Schwarze, M.; Kleemann, H.; Krammer, M.; Zojer, K.; Vandewal, K.; Leo, K. Elementary steps in electrical doping of organic semiconductors. Nat Commun 2018, 9, 1182. 
(3) Warren, R.; Privitera, A.; Kaienburg, P.; Lauritzen, A. E.; Thimm, O.; Nelson, J.; Riede, M. K. Controlling energy levels and Fermi level en route to fully tailored energetics in organic semiconductors. Nat Commun 2019, 10, 5538.

(4) Jacobs, I. E.; Moule, A. J. Controlling Molecular Doping in Organic Semiconductors. Adv. Mater. 2017, 29.

(5) Lussem, B.; Keum, C. M.; Kasemann, D.; Naab, B.; Bao, Z.; Leo, K. Doped Organic Transistors. Chem. Rev. 2016, 116, 13714-13751.

(6) B., L.; M., R.; K., L. Doping of organic semiconductors. physica status solidi (a) 2013, 210, 9-43.

(7) Walzer, K.; Maennig, B.; Pfeiffer, M.; Leo, K. Highly Efficient Organic Devices Based on Electrically Doped Transport Layers. Chem. Rev. 2007, 107, 1233-1271.

(8) Yoo, S. J.; Kim, J. J. Charge transport in electrically doped amorphous organic semiconductors. Macromol. Rapid Commun. 2015, 36, 984-1000.

(9) Jha, A.; Duan, H. G.; Tiwari, V.; Thorwart, M.; Miller, R. J. D. Origin of poor doping efficiency in solution processed organic semiconductors. Chem Sci 2018, 9, 4468-4476.

(10) Gasonoo, A.; Kim, J.-M.; Yoo, S.-J.; Cho, Y.-J.; Lee, J.-H.; Choi, Y.; Kim, J.-J.; Lee, J.-H. Analysis of the charge transfer and separation in electrically doped organic semiconductors by electron spin resonance spectroscopy. Org. Electron. 2019, 67, 242-246.

(11) Tietze, M. L.; Pahner, P.; Schmidt, K.; Leo, K.; Lüssem, B. Doped Organic Semiconductors: Trap-Filling, Impurity Saturation, and Reserve Regimes. Adv. Funct. Mater. 2015, 25, 2701-2707.

(12) Mendez, H.; Heimel, G.; Winkler, S.; Frisch, J.; Opitz, A.; Sauer, K.; Wegner, B.; Oehzelt, M.; Rothel, C.; Duhm, S.; Tobbens, D.; Koch, N.; Salzmann, I. Charge-transfer crystallites as molecular electrical dopants. Nat Commun 2015, 6, 8560.

(13) Salzmann, I.; Heimel, G.; Duhm, S.; Oehzelt, M.; Pingel, P.; George, B. M.; Schnegg, A.; Lips, K.; Blum, R. P.; Vollmer, A.; Koch, N. Intermolecular hybridization governs molecular electrical doping. Phys. Rev. Lett. 2012, 108, 035502.

(14) Wegner, B.; Grubert, L.; Dennis, C.; Opitz, A.; Röttger, A.; Zhang, Y.; Barlow, S.; Marder, S. R.; Hecht, S.; Müllen, K.; Koch, N. Predicting the yield of ion pair formation in molecular electrical doping: redox-potentials versus ionization energy/electron affinity. J. Mater. Chem. C 2019, 7, 13839-13848.

(15) Li, J.; D'Avino, G.; Pershin, A.; Jacquemin, D.; Duchemin, I.; Beljonne, D.; Blase, X. Correlated electron-hole mechanism for molecular doping in organic semiconductors. Physical Review Materials 2017, 1,025602.

(16) Kim, J.-M.; Yoo, S.-J.; Moon, C.-K.; Sim, B.; Lee, J.-H.; Lim, H.; Kim, J. W.; Kim, J.-J. N-Type Molecular Doping in Organic Semiconductors: Formation and Dissociation Efficiencies of a Charge Transfer Complex. J. Phys. Chem. C 2016, 120, 9475-9481.

(17) Li, J.; D'Avino, G.; Pershin, A.; Jacquemin, D.; Duchemin, I.; Beljonne, D.; Blase, X. Correlated electron-hole mechanism for molecular doping in organic semiconductors. Physical Review Materials 2017, 1 .

(18) Schwarze, M.; Gaul, C.; Scholz, R.; Bussolotti, F.; Hofacker, A.; Schellhammer, K. S.; Nell, B.; Naab, B. D.; Bao, Z.; Spoltore, D.; Vandewal, K.; Widmer, J.; Kera, S.; Ueno, N.; Ortmann, F.; Leo, K. Molecular parameters responsible for thermally activated transport in doped organic semiconductors. Nature Materials 2019, 18, 242-248. 
(19) Schwarze, M.; Tress, W.; Beyer, B.; Gao, F.; Scholz, R.; Poelking, C.; Ortstein, K.; Günther, A. A.; Kasemann, D.; Andrienko, D.; Leo, K. Band structure engineering in organic semiconductors. Science 2016, 352, 1446-1449.

(20) Warren, P. R.; Hardigree, J. F. M.; Lauritzen, A. E.; Nelson, J.; Riede, M. Tuning the ambipolar behaviour of organic field effect transistors via band engineering. AIP Advances 2019, 9, 035202.

(21) D’Avino, G.; Muccioli, L.; Castet, F.; Poelking, C.; Andrienko, D.; Soos, Z. G.; Cornil, J.; Beljonne, D. Electrostatic phenomena in organic semiconductors: fundamentals and implications for photovoltaics. J. Phys.: Condens. Matter 2016, 28, 433002.

(22) Blase, X.; Attaccalite, C.; Olevano, V. First-principles \$mathit $\{\mathrm{GW}\} \$$ calculations for fullerenes, porphyrins, phtalocyanine, and other molecules of interest for organic photovoltaic applications. Phys. Rev. B 2011, 83, 115103.

(23) Li, J.; D'Avino, G.; Duchemin, I.; Beljonne, D.; Blase, X. Accurate description of charged excitations in molecular solids from embedded many-body perturbation theory. Phys. Rev. B 2018, 97, 035108.

(24) da Silva Filho, D. A.; Coropceanu, V.; Gruhn, N. E.; de Oliveira Neto, P. H.; Brédas, J.-L. Intramolecular reorganization energy in zinc phthalocyanine and its fluorinated derivatives: a joint experimental and theoretical study. Chem. Commun. 2013, 49, 6069-6071.

(25) Brendel, M.; Krause, S.; Steindamm, A.; Topczak, A. K.; Sundarraj, S.; Erk, P.; Höhla, S.; Fruehauf, N.; Koch, N.; Pflaum, J. The Effect of Gradual Fluorination on the Properties of FnZnPc Thin Films and FnZnPc/C60 Bilayer Photovoltaic Cells. Adv. Funct. Mater. 2015, 25, 1565-1573.

(26) Li, J.; Duchemin, I.; Roscioni, O. M.; Friederich, P.; Anderson, M.; Da Como, E.; Kociok-Köhn, G.; Wenzel, W.; Zannoni, C.; Beljonne, D.; Blase, X.; D'Avino, G. Host dependence of the electron affinity of molecular dopants. Materials Horizons 2019, 6, 107-114.

(27) D’Avino, G.; Muccioli, L.; Zannoni, C.; Beljonne, D.; Soos, Z. G. Electronic Polarization in Organic Crystals: A Comparative Study of Induced Dipoles and Intramolecular Charge Redistribution Schemes. Journal of Chemical Theory and Computation 2014, 10, 49594971.

(28) Armin, A.; Stoltzfus, D. M.; Donaghey, J. E.; Clulow, A. J.; Nagiri, R. C. R.; Burn, P. L.; Gentle, I. R.; Meredith, P. Engineering dielectric constants in organic semiconductors. J. Mater. Chem. C 2017, 5, 3736-3747.

(29) D’Avino, G.; Duhm, S.; Della Valle, R. G.; Heimel, G.; Oehzelt, M.; Kera, S.; Ueno, N.; Beljonne, D.; Salzmann, I. Electrostatic Interactions Shape Molecular Organization and Electronic Structure of Organic Semiconductor Blends. Chem. Mater. 2020, 32, 1261-1271.

(30) Castet, F.; D'Avino, G.; Muccioli, L.; Cornil, J.; Beljonne, D. Charge separation energetics at organic heterojunctions: on the role of structural and electrostatic disorder. PCCP 2014, 16, 20279-20290.

(31) D’Avino, G.; Mothy, S.; Muccioli, L.; Zannoni, C.; Wang, L.; Cornil, J.; Beljonne, D.; Castet, F. Energetics of Electron-Hole Separation at P3HT/PCBM Heterojunctions. J. Phys. Chem. C 2013, 117, 12981-12990.

(32) Athanasopoulos, S.; Tscheuschner, S.; Bässler, H.; Köhler, A. Efficient Charge Separation of Cold Charge-Transfer States in Organic Solar Cells Through Incoherent Hopping. The Journal of Physical Chemistry Letters 2017, 8, 2093-2098.

(33) Idé, J.; Méreau, R.; Ducasse, L.; Castet, F.; Bock, H.; Olivier, Y.; Cornil, J.; Beljonne, D.; D’Avino, G.; Roscioni, O. M.; Muccioli, L.; Zannoni, C. Charge Dissociation at 
Interfaces between Discotic Liquid Crystals: The Surprising Role of Column Mismatch. J. Am. Chem. Soc. 2014, 136, 2911-2920. 